\title{
Unusual predominance of maintenance DNA methylation in Spirodela polyrhiza
}

\author{
Alex Harkess ${ }^{1 *}$, Adam J. Bewick ${ }^{2 *}$, Zefu Lu², Paul Fourounjian ${ }^{3}$, Joachim Messing ${ }^{3 \dagger}$, Todd P. \\ Michael $^{4}$, Robert J. Schmitz ${ }^{2, \wedge}$, Blake C. Meyers ${ }^{1,5, \wedge}$ \\ ${ }^{1}$ Donald Danforth Plant Science Center, St Louis MO 63132 \\ ${ }^{2}$ Department of Genetics, University of Georgia, Athens GA 30602 \\ ${ }^{3}$ Waksman Institute of Microbiology, Rutgers University, New Brunswick NJ 08901 \\ ${ }^{4}$ Salk Institute for Biological Studies, La Jolla CA 92037 \\ ${ }^{5}$ University of Missouri - Columbia, Division of Plant Sciences, Columbia, MO 65211, USA \\ *These authors contributed equally \\ $\dagger$ Deceased. \\ ${ }^{\wedge}$ Corresponding Authors: Blake C. Meyers, Robert J. Schmitz.
}

\begin{abstract}
5-methylcytosine $(5 \mathrm{mC})$ is a modified base often described as necessary for the proper regulation of genes and transposons and for the maintenance of genome integrity in plants. However, the extent of this dogma is limited by the current phylogenetic sampling of land plant species diversity. Here, we report that a monocot plant, Spirodela polyrhiza, has lost CG gene body methylation, genome-wide $\mathrm{CHH}$ methylation, and the presence or expression of several genes in the highly conserved RNA-directed DNA methylation (RdDM) pathway. It has also lost the $\mathrm{CHH}$ methyltransferase CHROMOMETHYLASE 2. Consequently, the transcriptome is depleted of 24-nucleotide, heterochromatic, small interfering RNAs that act as guides for the deposition of $5 \mathrm{mC}$ to RdDM-targeted loci in all other currently sampled angiosperm genomes. Although the genome displays low levels of genome-wide $5 \mathrm{mC}$ primarily at LTR retrotransposons, $\mathrm{CG}$ maintenance methylation is still functional. In contrast, $\mathrm{CHG}$ methylation is weakly maintained even though $\mathrm{H} 3 \mathrm{~K} 9 \mathrm{me} 2$ is present at loci dispersed throughout the euchromatin and highly enriched at regions likely demarcating pericentromeric regions. Collectively, these results illustrate that $S$. polyrhiza is maintaining CG and CHG methylation mostly at repeats in the absence of small RNAs. $S$. polyrhiza reproduces rapidly through clonal propagation in aquatic environments, which we hypothesize may enable low levels of maintenance methylation to persist in large populations.
\end{abstract}

Significance Statement: DNA methylation is a widespread chromatin modification that is regularly found in all plant species. By examining one aquatic duckweed species, Spirodela polyrhiza, we find that it has lost highly conserved genes involved in methylation of DNA at sites often associated with repetitive DNA, and within genes, however DNA methylation and heterochromatin is maintained during cell division at other sites. Consequently, small RNAs that normally guide methylation to silence repetitive DNA like retrotransposons are diminished. Despite the loss of a highly conserved methylation pathway, and the reduction of small RNAs that normally target repetitive DNA, transposons have not proliferated in the genome, perhaps due in part to the rapid, clonal growth lifestyle of duckweeds.

\section{Introduction}


Evolutionary theory predicts that asexual populations should become less fit over time due to an irreversible accumulation of deleterious alleles (1). Duckweeds are perhaps the most striking counter-example in plants, given their cosmopolitan distribution and ability to survive in diversely harsh environments (2). Duckweed is a common name for all 36 species in the Lemnaceae family of monocots, divided across five genera: Spirodela, Lemna, Landoltia, Wolffia, and Wolffiella (Figure 1A). Most duckweed species rarely flower, instead reproducing primarily by rapid, clonal reproduction that occurs at one of the fastest rates in any angiosperm (3). The largest duckweed species Spirodela polyrhiza ( 1 cm wide) intriguingly has the smallest genome size ( $\sim 158$ megabases) (3-5), and several genome assemblies consistently annotate fewer than 20,000 genes (6). Compared to the Arabidopsis thaliana genome that is roughly the same total genome size, $S$. polyrhiza has nearly $25 \%$ fewer genes. Without much meiotic recombination through sexual reproduction, and fewer genes for selection to act upon, epigenetic variation could instead be a promising mechanism to explain the global success of clonal duckweeds (7).

Sexual reproduction in plants is often accompanied by widespread genome-wide reinforcement of DNA methylation with localized epigenetic reprogramming in gametes (8-10). This results in patterns of both stable DNA methylation inheritance and infrequent spontaneous epialleles (11). However, nearly $60 \%$ of global crops can be bred through clonal propagation (12), highlighting the need to illustrate how epigenetics can be used to improve plant breeding efforts. Cytosine DNA methylation or 5-methylcytosine $(5 \mathrm{mC})$, is found in species spanning the flowering plant phylogeny (13). As the number and phylogenetic diversity of plant genomes and DNA methylomes increases, so does the observed diversity of $5 \mathrm{mC}$ levels, specificity and DNA methyltransferase enzymes. 5mC DNA methylation in plants occurs at three major sequence contexts, each of which require different sets of enzymes to function: $\mathrm{CG}, \mathrm{CHG}$, and $\mathrm{CHH}$ (where $\mathrm{H}=\mathrm{A}, \mathrm{C}, \mathrm{T}$ ). Methylation at these different contexts is established by both de novo and maintenance methyltransferase enzymes. DNA methylation at CG and CHG sites is typically symmetrical across the Watson and Crick strands, whereas DNA methylation at CHH sites is asymmetrical. The observed symmetry is due to the mechanisms by which $5 \mathrm{mC}$ is maintained after DNA replication. Methylation at CG sites relies on the maintenance methyltransferase METHYLTRANSFERASE 1 (MET1) (14-16), whereas maintenance of methylation at CHG sites relies on a positive feedback loop between dimethylation of lysine 9 on histone 3 (H3K9me2) and CHROMOMETHYLASE 3 (CMT3) (17-20). DNA methylation at CHH sites is asymmetrical and is further classified into CWA (where $\mathrm{W}=\mathrm{A}$ or $\mathrm{T}$ ) and non-CWA, based on targeting by CHROMOMETHYLASE 2 (CMT2) or by 24-nt siRNAs and DOMAINS REARRANGED METHYLTRANSFERASE 2 (DRM2), which are associated with the RNA-directed DNA methylation $(\operatorname{RdDM})$ pathway, respectively $(21,22)$.

Variation in DNA methylation has been connected to pathogen response (23), temperature tolerance (24), and geography (25), which could be crucial attributes for clonal duckweeds given their reduced ability generate genetic variation through recombination. Intriguingly, the duckweed $S$. polyrhiza displays particularly low levels of 5mC, with evidence that low DNA methylation levels are likely related to its small genome size with the low amounts of repetitive DNA (26). However, the mechanisms underlying this variation in DNA methylation are unknown (6). Here we dissect 5mC DNA methylation patterns, histone modifications, small RNAs, and the genes that control major methylation pathways in S. polyrhiza. We discover that S. polyrhiza has lost the activity of key, canonical DNA methylation and small RNA pathway 
genes that consequently diminish gene body methylation, the RNA-directed DNA methylation pathway, and genome-wide $\mathrm{CHH}$ methylation.

\section{Results and Discussion}

To test if low levels of $5 \mathrm{mC}$ might be a conserved feature across the diversity of $S$. polyrhiza, we performed whole genome bisulfite sequencing across two different genotypes (lines 7498 and 9509) (Supplemental Table 1). Both genotypes show similar patterns: roughly $10 \%$ of the CG sites in the genome are methylated (Figure 1B). Fewer than $3.28 \%$ and $3.67 \%$ of CHG and $0.0065 \%$ and $0.035 \% \mathrm{CHH}$ sites are significantly methylated (Figure 1B). In $S$. polyrhiza, mCG and some mCHG (specifically CAG and CTG) are symmetrically maintained through equal DNA methylation on the Watson and Crick strands, which are normal features of maintenance methylation (Figure 1C). However, the maintenance of $\mathrm{mCHG}$ in S. polyrhiza is weak in comparison to other species that possess a functional CMT3 (13). CG methylation is present at small clusters of Long Terminal Repeat (LTR) retrotransposons in the genome (Figure 1D), but $\mathrm{CHH}$ methylation which is normally enriched in repetitive elements like LTR retrotransposons (27), is absent (Figure 1C-D).
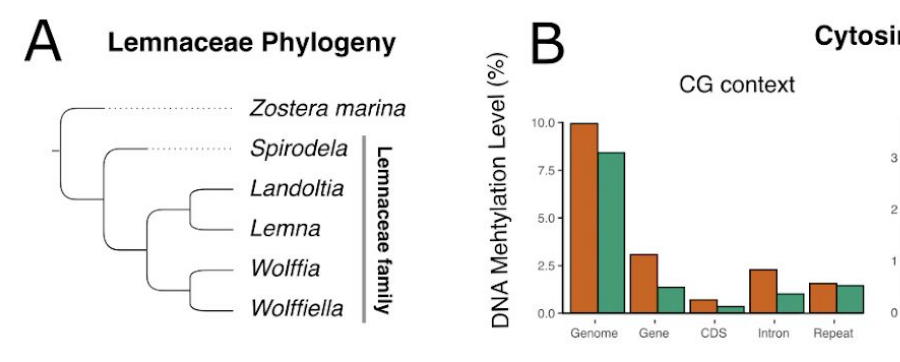

Cytosine Methylation Patterns Across Features

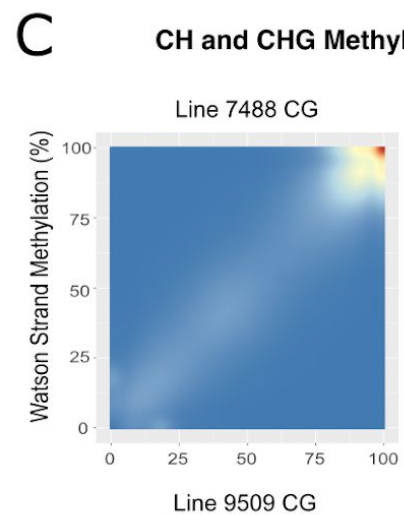

lation Symmetry
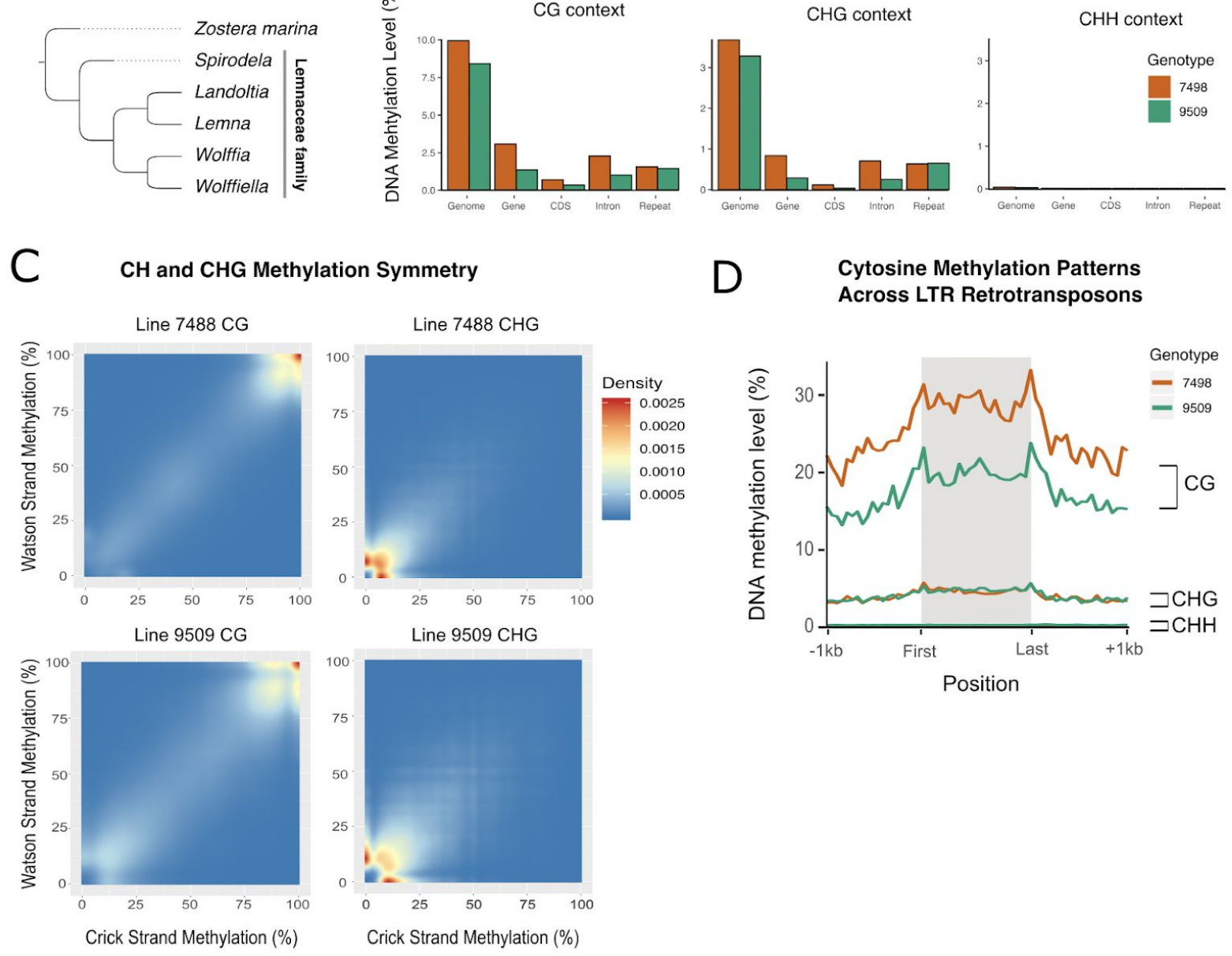

Figure 1: A) A condensed species tree of the Lemnaceae family, with five genera: Spirodela, Landoltia, Lemna, Wolffia, Wolffiella. B) DNA methylation level for whole genome, genes, 
$\mathrm{CDS}$, introns, and repeats, across $\mathrm{CG}, \mathrm{CHG}$, and $\mathrm{CHH}$ site contexts, in two genotypes of Spirodela polyrhiza. C) Site-wise methylation symmetry of CG and CHG on Watson and Crick strands. D) DNA methylation levels across LTR retrotransposons, across CG, CHG, and CHH sites, in two genotypes of Spirodela polyrhiza.

DNA methylation at some CHH sites is guided by small RNAs (sRNAs) generated through the RdDM pathway (28) (Figure 2A), so small RNA (sRNA) sequence reads were generated for both $S$. polyrhiza lines to test for functional defects in the pathway (Supplemental Table 2). Both lines display a distinct lack of 24-nucleotide (nt), heterochromatic siRNAs (het-siRNAs) which are typically the most abundant size class of angiosperm $\operatorname{sRNAs}(29,30)$ (Figure 2B). The highly conserved, canonical RdDM pathway produces these $24 \mathrm{nt}$ het-siRNAs via DICER-LIKE 3 (DCL3) processing of an RNA Polymerase IV (Pol IV)-derived double-stranded RNA $(31,32)$. These DCL3-derived sRNAs are loaded into ARGONAUTE 4 (AGO4) and guided to their sites of action (Figure 2A) $(28,33,34)$. Due to the reduction of 24 nt het-siRNAs, available whole plant mRNA-seq data was mined for evidence of the expression of RdDM-related genes (Figure 2C). DCL3 is present in the genome as a seemingly full-length sequence with no in-frame stop codons, but no gene expression was detectable (FPKM $<1)$. DCL3 expression is also not detected under various growth and stress conditions in S. polyrhiza, including copper, kinetin, nitrate and sucrose additions (35) (Supplemental Fig. 1). The DCL3 upstream region is short (fewer than $200 \mathrm{nt}$ ), and possibly interrupted by another gene, which may entirely disrupt DCL3 gene activity (Supplemental Fig. 2).

Given an absence of detectable DCL3 expression (Figure 2C), we investigated the presence and expression of orthologs of other plant Dicer-like genes (DCL1, DCL2, DCL4). $D C L 1$, which functions in microRNA (miRNA) production, is expressed and produces many conserved miRNAs, indicating it functions normally (36). However, DCL2, which functions largely in viral defense, is missing from the $S$. polyrhiza genome (37) (Supplemental Fig. 3). DCL4, which generates 21-nt siRNAs, is present in the genome and expressed (38-40). DCL5, which is implicated in phased siRNA production in maize (41) and has a role in flower fertility (42), is also not present in the genome (30) (Supplemental Fig. 3). In addition to DCL2 and $D C L 3$, there was no detectable expression for $A G O 4$, nor the genes encoding the two major catalytic subunit genes of the Pol IV complex (NRPDI and NRPE1) that transcribe single-stranded RNA precursors from RdDM regions and are required for siRNA and methylation-dependent heterochromatin formation (43) (Figure 2C). CMT3 and MET1 are expressed in $S$. polyrhiza, consistent with their roles in the maintenance of $\mathrm{CG}$ and $\mathrm{CHG}$ methylation in the $A$. thaliana genome (Figure 2C). We next tested whether the lack of expression of some RdDM genes is a conserved phenomenon across some or all duckweed species in the Lemnaceae family. De novo transcriptome assemblies of publicly available whole plant RNA-seq data for species from two genera of duckweeds, Landoltia punctata and Lemna minor (Figure 1A), were interrogated for Dicer-like gene expression. In both L. punctata and $L$. minor, de novo transcripts were assembled for DCL1 and DCL4, however there were no assemblies with BLASTX hits (1e-10) to DCL2 and DCL3. Although whole genome assemblies of species representing all five genera of duckweeds will be needed to definitively test this hypothesis, these data suggest that the expression loss of DCL2 and DCL3, possibly leading to the loss of canonical RdDM, may be a widespread phenomenon across several genera of the Lemnaceae family (Figure 1A). 


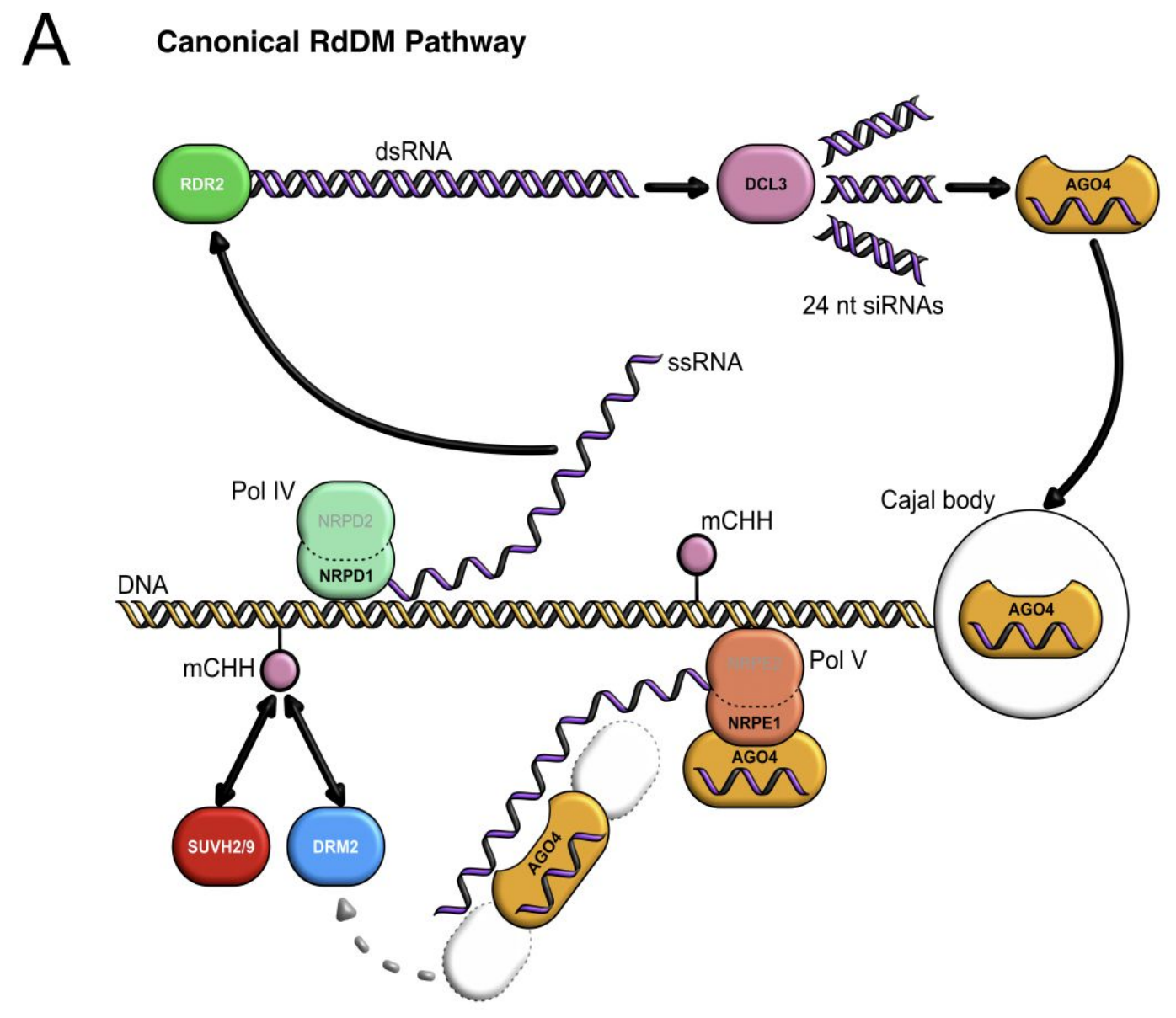

B Small RNA Sequencing Size Distribution

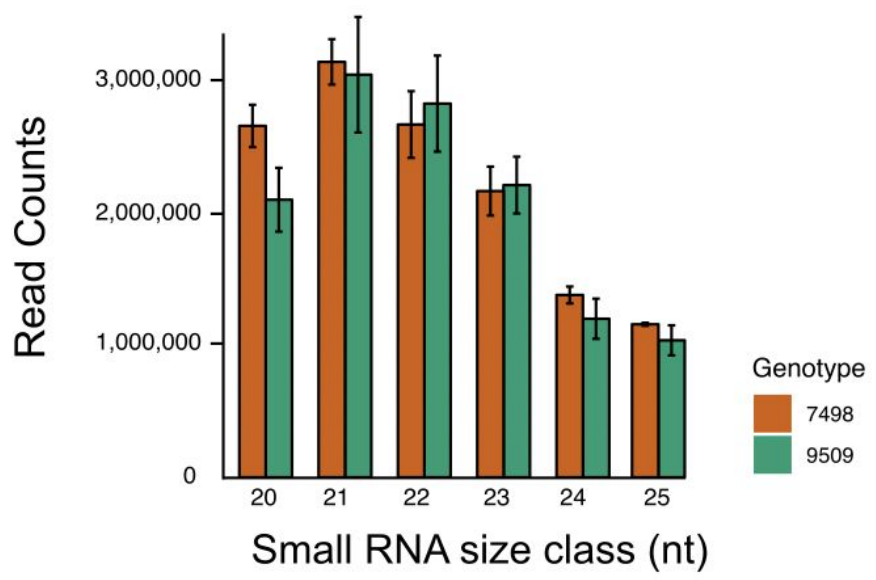

C RdDM Pathway Gene Expression

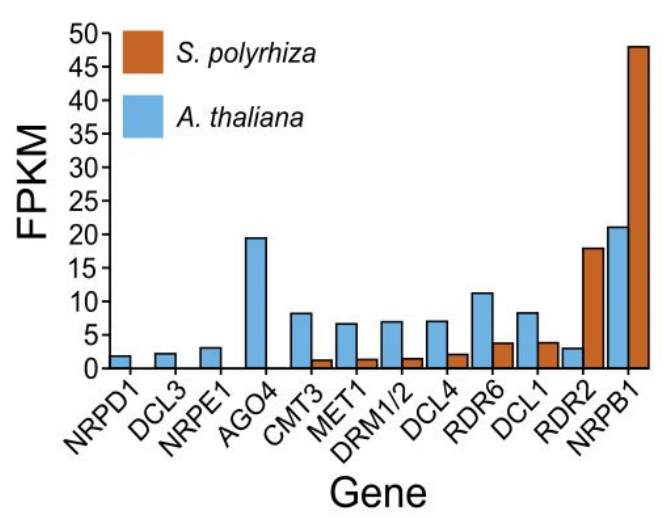

Figure 2: A) Diagram of the canonical RNA-directed DNA methylation (RdDM) pathway in plants. RNA Polymerase IV (Pol IV) transcribes a single-stranded RNA (ssRNA) which is 
converted to a double-stranded RNA (dsRNA) by RNA-DIRECTED RNA POLYMERASE 2 (RDR2). DICER-LIKE 3 (DCL3) then cleaves those dsRNA products into 24 nucleotide small RNA (sRNA) products. One strand of each sRNA is loaded into ARGONAUTE 4 (AGO4), and the AGO-sRNA complex binds to complementary RNA sequences transcribed by RNA Polymerase V (Pol V), guided by interaction with SUVH2 and SUVH9. DOMAINS REARRANGED METHYLTRANSFERASE 2 (DRM2) is then recruited, which guides methylation of DNA at those sites. B) The distribution of small RNA sequence read abundance between 20-25 nucleotides in two genotypes of S. polyrhiza. C) Gene expression in S. polyrhiza line 9509 and $A$. thaliana measured by RNA-seq for several RdDM and methylation-related genes.

Although RdDM is one route to forming $\mathrm{CHH}$ methylation, an RdDM-independent mechanism is through the action of CHROMOMETHYLASE 2 (CMT2), a plant-specific DNA methyltransferase that is highly conserved across angiosperms $(21,44,45)$ (Figure 3A). CHH sites targeted by the RdDM pathway typically show enrichment at all contexts $(21,46)$, which $S$. polyrhiza does not exhibit (Figure 3A). In A. thaliana, CHH methylation deposited via CMT2 can be distinguished from RdDM-targeted sites given that they show an enrichment of CWA methylation $(\mathrm{W}=\mathrm{A}$ or $\mathrm{T})$ relative to other contexts $(21,46)$ and they are enriched at regions possessing H3K9me2 (44). However, a CMT2 homolog is missing from the S. polyrhiza genome (lines 7498 and 9509) (Figure 3B). As expected given the loss of CMT2, there is no enrichment of CWA methylation in either genotype (Figure 3C). There is a low level of CWG methylation in both lines, though (Figure 3C). CWG methylation is dependent on CMT3 (21), which is present and expressed (Figure 2C). Across the global range of $A$. thaliana, there is extensive variation at the $C M T 2$ locus including a non-functional $c m t 2$ allele that is associated with reduced genome-wide $\mathrm{CHH}$ methylation, but also the benefit of an increased tolerance to heat stress $(24,47)$. Given that $S$. polyrhiza is globally distributed and thrives in a variety of climates and stresses, increased genotyping and phenotyping of diverse populations may reveal similar patterns of methylation-sensitive phenotypes. Intriguingly, CMT2 is missing in the maize genome (48), but also missing from the aquatic seagrass Zostera marina genome assemblies and annotations (Figure 3B), suggesting that CMT2 loss may be a shared feature that has evolved in multiple aquatic plants in the Alismatales order. Despite a lack of expression of key RdDM genes and sRNAs that normally function to target repetitive DNA, there has not been a recent detectable expansion of LTR retrotransposons in the $S$. polyrhiza genomes (Figure 3D) $(3,26)$, nor are they methylated in the typical CHH context (Figure 1D). Specifically, only 3/1114 $(0.003 \%)$ and 6/1510 (0.004\%) LTR retrotransposons are enriched for $\mathrm{CHH}$ methylation in 7498 and 9509 genomes, respectively, and likely false positives (48). 

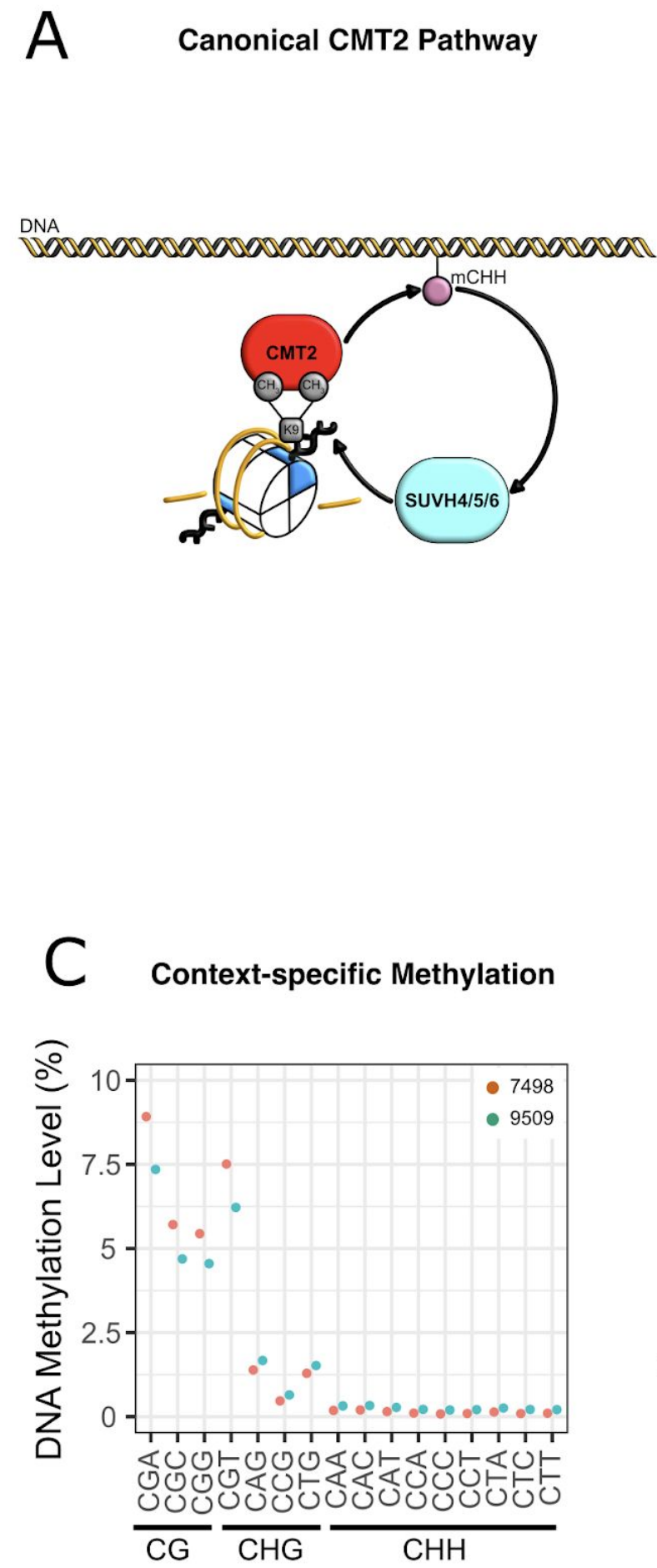

\section{B CMT2 Presence Across Monocots}

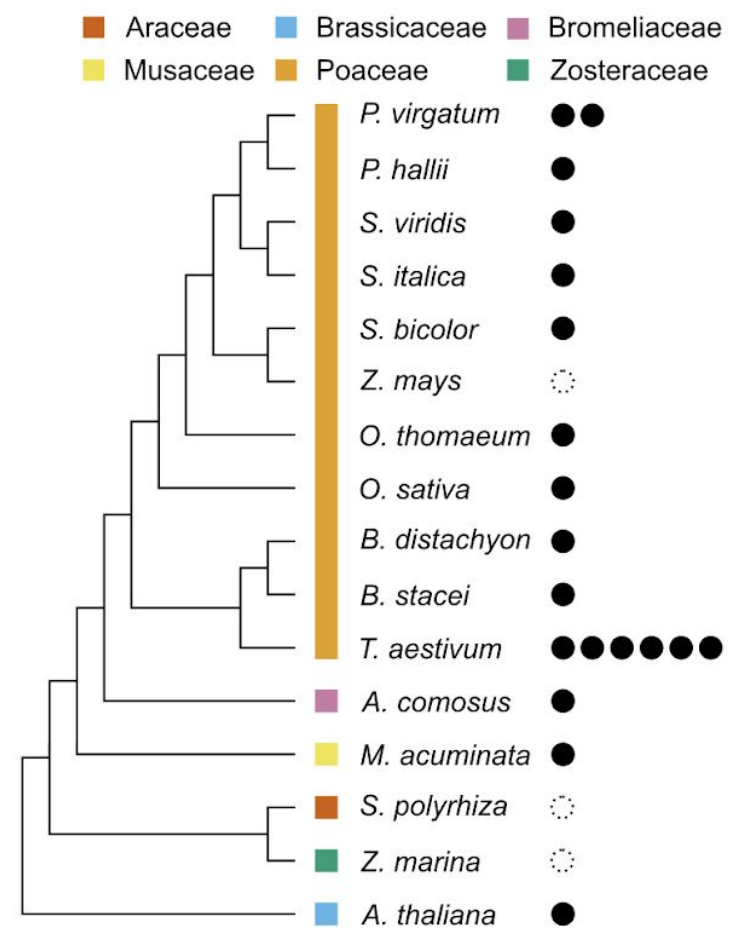

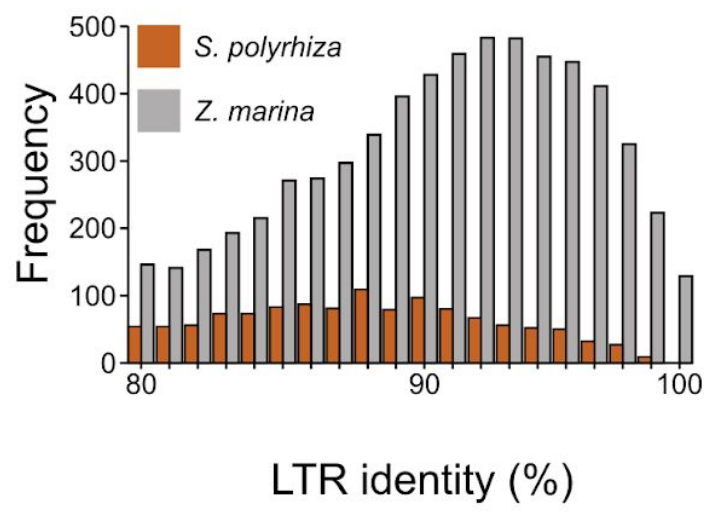

Figure 3: A) Diagram of the canonical CHROMOMETHYLASE 2 (CMT2) pathway. B) Presence (filled circles) and absence (empty circles) of CMT2 homologs in genomes across the monocots. C) Genome-wide DNA methylation of two genotypes of $S$. polyrhiza split into all possible contexts. D) Relative LTR retrotransposon insertion timings between S. polyrhiza and Zostera marina, based on LTR percent identity comparisons. 
The loss of CHH methylation, 24 nt het-siRNAs, and CMT2 suggests that the abundance of heterochromatin may also be low. Cao et al. (49) made an observation using $5 \mathrm{mC}$ and histone 3 lysine 9 di-methylated (H3K9me2) immunostaining, a common histone modification in heterochromatic regions of the genome (50), that $S$. polyrhiza and four other genera of the Lemnaceae lack strong signals of concentrated heterochromatic blocks of DNA. H3K9 methylation mediates $\mathrm{CHG}$ and $\mathrm{CHH}$ methylation through the action of CMT3 and CMT2, respectively (44). To test if the CMT2 loss and the weak levels of CHG methylation is tied to a reduction of $\mathrm{H} 3 \mathrm{~K} 9$ methylation in $S$. polyrhiza, we performed chromatin immuno-precipitation sequencing (ChIP-seq) of H3K9me2 (Supplemental Table 3). H3K9me2 is sparsely distributed throughout the euchromatic chromosome arms and shows a discrete enrichment of a large domain within each chromosome (Fig 4A). These relatively larger domains of H3K9me2 are approximately 400-600 $\mathrm{kb}$ and presumably reflect the pericentromeric regions similar to observations in other angiosperms like $A$. thaliana (Fig 4A). H3K9me2 occupies $\sim 15 \%$ of the line 9509 genome. In the 9509 genome, 746/1,510 (49.40\%) LTR retrotransposon annotations overlap H3K9me2 (Fisher's Exact Test, p < 0.001; Supplemental Table 4, Supplemental Fig, 4). Overall, H3K9me2 and heterochromatin appears normal in S. polyrhiza, especially when considering the small genome size split into 20 chromosomes.

Maintenance of DNA methylation at heterochromatin is associated with the establishment and maintenance of gene body DNA methylation (gbM) (46, 51-53). It is characterized by an enrichment of CG DNA methylation between the transcription start site and transcription termination site of genes $(45,54)$. Genes with gbM are typically moderately expressed throughout all tissues, long and exhibit low rates of nucleotide substitutions compared to non-gbM genes $(55,56)$. We blindly quantified CG methylation in coding regions of each gene, only accounting for the number of methylated CG sites, total CG sites, and read coverage (Figure 4C). In S. polyrhiza, this resulted in 541 and 520 putative gbM genes in lines 7498 and 9509 , respectively, or $2-3 \%$ of the total gene annotation set. Comparing the two putative gbM gene sets, 118 genes overlap between the two genomes (blastp 1e-40), which is unexpected as gbM genes are often highly conserved $(53,55)$ (Figure 4B). These results are similar to another species that has lost gbM, Eutrema salsugineum, where roughly 500 genes were bioinformatically detected as having gbM signatures using similar methodology (52). This result is likely driven by a similar false positive rate of gbM gene detection in both species, as well as transposon misannotation, and that like E. salsugineum, gbM has been lost in S. polyrhiza. 


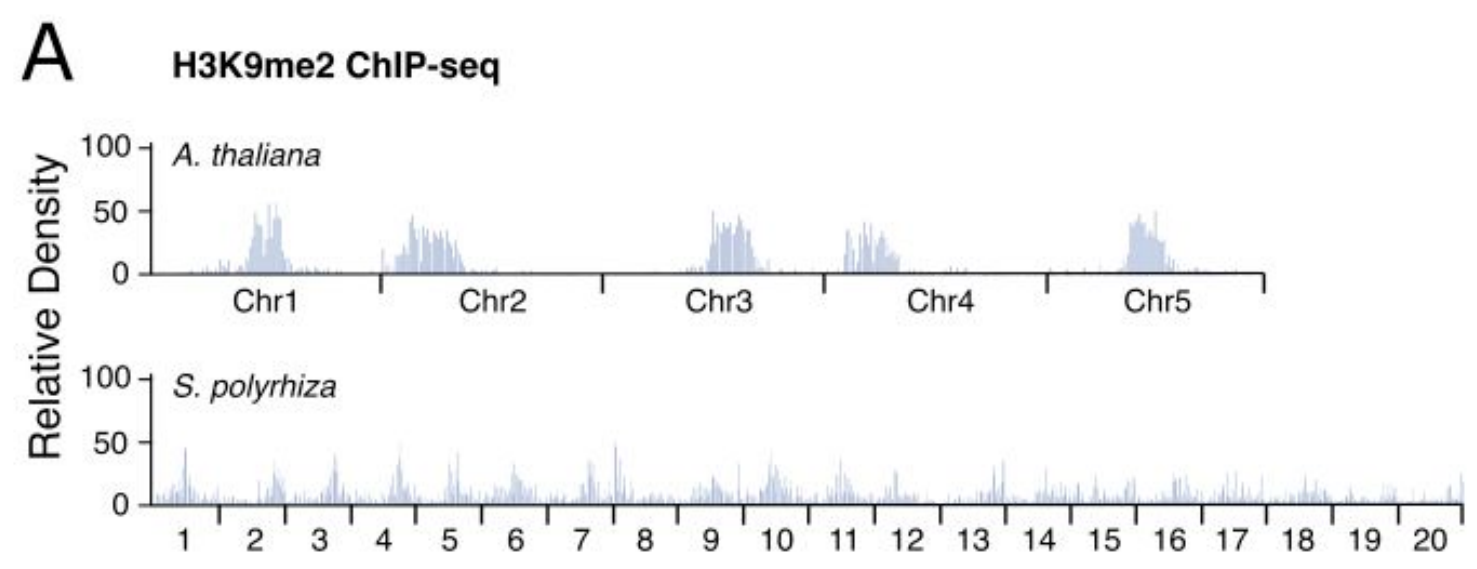

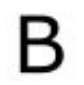

\section{CG Gene Body Methylation}
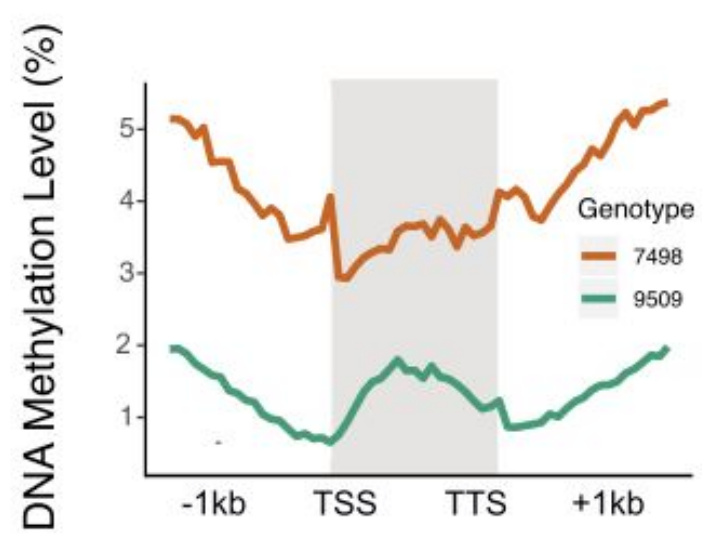

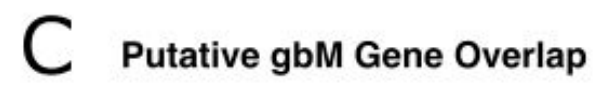

Figure 4: A) Distribution of H3K9me2 ChIP-seq peaks in A. thaliana and S. polyrhiza line 9509. B) Weighted gene body methylation plotted along CDS regions spanning from Transcription Start Site (TSS) to Transcription Termination Site (TTS), plus or minus 1 kilobase. C) The overlap of blindly calling putative gene body methylated genes in two genotypes of S. polyrhiza.

The faithful establishment and maintenance of gbM is tied to a self-reinforcing feedback loop that relies on the interplay between CMT3 and $\mathrm{H} 3 \mathrm{~K} 9 \mathrm{me} 2(17,46,53,57)$. This is further supported by studies in $A$. thaliana whereby mutants that result in a loss of maintenance of heterochromatin lead to ectopic activity of CMT3 in gbM genes (58-60). In S. polyrhiza, we show that the CMT3/H3K9me2 feedback loop is weak in comparison to other angiosperms, even though $\mathrm{H} 3 \mathrm{~K} 9 \mathrm{me} 2$ has a typical distribution throughout the genome. Therefore, it is possible that CMT3 activity is impaired, which leads to a weakly functioning feedback loop in S. polyrhiza and the loss of gbM. These results are consistent with proposed models from Wendte et al. (46) and Inigaki and Kakutani (51), in which CMT3 and H3K9me2 work coordinately to establish de novo gbM.

Ecological life history and developmental traits may strongly influence genome-wide patterns of DNA methylation and inheritance, especially relating to the suppression of 
transposon expansion over time. $S$. polyrhiza primarily reproduces via rapid clonal propagation rather than by flower production and sex (61), though some low frequency instances of flowering have been reported $(62,63)$. The methylomes of other clonally propagated species (Eucalyptus grandis, Fragaria vesca, Manihot esculenta, Theobroma cacao, and Vitis vinifera) possess $\mathrm{mCHH}$, although the levels are lower than non-clonally reproducing angiosperms $(13,64)$. This suggests that $\mathrm{CHH}$ reinforcement is linked to sexual reproduction, but isn't necessary for transposon silencing as clonally propagated species rely more on maintenance DNA methylation. Resequencing of globally distributed $S$. polyrhiza accessions reveals very little per-site genetic diversity within the species, a low recombination rate, and weak purifying selection, but still a large effective population size $\left(N_{e}\right)(65,66)$. Few transposons exist in the $\sim 150 \mathrm{Mb}$ S. polyrhiza genome, but a high ratio of solo-LTRs to intact LTR retrotransposons (26) suggests that LTR excision is actively occurring despite weak purifying selection. Individuals with fewer transposons and the ability to excise them may have selective advantages in large populations (67), given that a deleterious transposon insertion is unlikely to propagate and fix in a large clonal population, possibly compensating for the lack of a canonical RdDM pathway.

Several alternative hypotheses could explain the lack of CHH methylation and $24 \mathrm{nt}$ het-siRNAs. DNA methylation and sRNA production may be limited to cell or tissue-specific regions, such as the developing meristematic region where daughter plantlets emerge. Tissue sampling for duckweeds is often performed on several individual plantlets combined, given the small size, which would reduce the detectable signal of tissue- or cell type-specific changes and require more precise excision or cell sorting techniques. Overall, S. polyrhiza displays a loss of CHH-type DNA methylation and heterochromatic siRNAs, which may be tied to its rapid asexual reproduction. Our work in S. polyrhiza demonstrates that reproductive success through rapid clonal propagation may benefit from the sacrifice of the RdDM and CMT2 pathways.

\section{Data Availability}

All raw small RNA, DNA methylation, and H3K9me2 ChIP-seq data is available at BioProject GSE161234. Epigenome browsers are available for S. polyrhiza line 7498

(http://epigenome.genetics.uga.edu/SchmitzLab-JBrowse/?data=spi_pol_7498) and for line 9509 (http://epigenome.genetics.uga.edu/SchmitzLab-JBrowse/?data=spi_pol 9509).

\section{Author contributions}

The study was conceived by AH, AJB, RJS, and BCM. AH and AJB performed genomic, phylogenetic and evolutionary analyses. AH, AJB, TM and PF performed gene expression and sRNA analyses, and AJB performed DNA methylation analyses. ZL generated and analyzed H3K9 ChIP data. PF and JM provided materials and assistance with expression analyses. AH and AJB wrote the article with contributions made by all authors.

\section{Materials and Methods}

\section{DNA methylation sequencing and sequence alignment}

Whole-genome bisulfite sequencing data were generated according to (68). Single-end short read libraries (150 bp) were aligned using the methylpy pipeline (69) to the S. polyrhiza 7498 and 9509 genomes. Methylpy calls programs for read processing and aligning: (i) reads were trimmed of sequencing adapters using Cutadapt (70), (ii) and then mapped to both a 
converted forward strand (cytosines to thymines) and converted reverse strand (guanines to adenines) using bowtie (71). Reads that mapped to multiple locations, and clonal reads were removed. The chloroplast genome (GenBank: JN160603.2) was used to estimate a rate of sodium bisulfite non-conversion.

\section{DNA methylation analyses}

DNA methylation levels were estimated as weighted DNA methylation, which is the total number of aligned DNA methylated reads divided by the total number of methylated plus un-methylated reads with a minimum coverage of at least 5 reads (72). Global weighted DNA methylation was estimated across the entire genome, within intergenic regions, transposons, genes (exons+introns), exons and introns. Additionally, the genome was divided into non-overlapping $50000 \mathrm{bp}$ windows, and weighted DNA methylation was estimated for each window.

For metaplots, the locus body - start-to-stop codon for genes and first to last bp for transposons - was divided into 20 proportional windows based on locus length. Within gene bodies only sequenced reads mapping to coding, exonic DNA were used. Additionally, $1000 \mathrm{bp}$ upstream and downstream were divided into 20 proportional windows. A single weighted DNA methylation value was calculated for each window across all loci.

For each gene, a binomial test with a Benjamini-Hochberg False Discovery Rate (FDR) correction was applied to determine enrichment of DNA methylation at the three sequence contexts (CG, $\mathrm{CHG}$, and $\mathrm{CHH}$ ). Only $\mathrm{CG}, \mathrm{CHG}$ and $\mathrm{CHH}$ sites found within coding, exonic sequences were considered. The weighted DNA methylation level of cytosines at $\mathrm{CG}, \mathrm{CHG}$ and $\mathrm{CHH}$ sites across all coding regions were used as the probability of success, respectively. Enrichment tests for gene body methylation were performed using code from (53), found at https://github.com/schmitzlab/Natural_variation in_DNA methylation homeostasis and the e mergence of epialleles.

To determine per-site methylation levels, the weighted DNA methylation level for each cytosine with $\geq 3$ reads of coverage was calculated. Additionally, DNA methylation levels of symmetrical cytosines $(\mathrm{CG}$ or $\mathrm{CWG}, \mathrm{W}=\mathrm{A} \mid \mathrm{T})$ with $\geq 3$ sequencing coverage were estimated for each strand (Watson and Crick). All plots were generated in R v3.2.4

(https://www.r-project.org/).

\section{sRNA and mRNA sequencing analysis}

sRNA sequencing reads were generated using whole plant total RNA isolated using TRI reagent and the Somagenics RealSeq-AC kit with $100 \mathrm{ng}$ of total RNA as input. Reads were adapter-trimmed with cutadapt v2.0 (70) with options " $-\mathrm{m} 15$

TGGAATTCTCGGGTGCCAAGG". Cleaned reads were aligned to the reference genome using bowtie with settings "-a -v 0" to only report end-to-end alignments with zero mismatches.

Raw mRNA-Seq reads from strain 9509 were retrieved from the Sequence Read Archive (SRR3090696), cleaned with Trimmomatic v0.32 with settings "ILLUMINACLIP:2:30:10 LEADING:3 TRAILING:3 SLIDINGWINDOW:4:15 MINLEN:50" and aligned to the reference genome with TopHat v2.1.1 with default settings other than "-i 25". Per-gene expression was calculated with Cufflinks v2.2.1 with default settings. 
ChIP was performed as previously described (73). Briefly, $1 \mathrm{~g}$ of fresh duckweed plantlets were crosslinked in $1 \%$ formaldehyde for $10 \mathrm{~min}$. Nuclei were then isolated and sonicated for $15 \mathrm{~min}$, twice. Histone-DNA complexes were pulled down with anti-H3K9me2 (Cell Signaling Technology antibody \#9753s). DNA was isolated and used to prepare ChIP-seq libraries with the TruSeq ChIP Library Preparation Kit (Illumina, IP-202-1012). Sequencing was performed on an Illumina NextSeq500 in Georgia Genomics and Bioinformatics Core (GGBC) in the University of Georgia.

Raw ChIP reads were trimmed for adapters and low-quality bases using Trimmomatic with the following options: reads were trimmed for TruSeq version 3 single-end adapters with a maximum of two seed mismatches, palindrome clip threshold of 30, and simple clip threshold of 10. Trimmed reads were mapped to the genome using bowtie1 with "-v 2 --best --strata -m 1" (71). Mapped reads were sorted using SAMtools (74) and then clonal duplicates were removed using picard (http://broadinstitute.github.io/picard/). Remaining reads were converted to BED format with Bedtools (75). H3K9me2 enriched regions were identified with MACS2 with parameter "--keep-dup all --broad" (76). Enrichment of H3K9me2 overlaps with LTR retrotransposons was tested using a Fisher's Exact Test implemented in bedtools v2.26.0.

\section{Phylogenetic analyses}

CHROMOMETHYLASE (CMT) protein sequences were obtained from (45), and additional sequences were identified in monocot species listed on Phytozome v12 (https://phytozome.jgi.doe.gov/pz/portal.html) using best blastp hit e-value $\leq 1 \mathrm{E}-06$ and bit score $\geq 200$ ) to $A$. thaliana CMT1 (AT1G80740.1), CMT2 (AT4G19020.1) and CMT3

(AT1G69770.1). Similarly, DICER-LIKE (DCL) homologs were identified in all monocot species listed on Phytozome v12 using best BLASTP hit to A. thaliana DCL1 (AT1G01040.2), DCL2 (AT3G03300.1), DCL3 (AT3G43920.2), and DCL4 (AT5G20320.1). Protein sequences were aligned using the program PASTA with default parameters. Following alignment, GBblocks was used to identify conserved amino acid positions. All parameters were kept at the default setting except $-\mathrm{b} 2=n 0.66$ where $n$ is the number of sequences and $-\mathrm{b} 5=\mathrm{h}$. BEAST v2.3.2 was used to estimate the phylogeny with a BLOSUM62 substitution matrix. The MCMC chain in BEAST was allowed to run until stationarity and convergence (ESS $\geq 200$ ) was reached, and was assessed using the program Tracer v1.6. A maximum clade credibility tree was generated from the posterior distribution of trees with the burn-in removed using the program TreeAnnotator v2.3.2. Finally, the program FigTree (http://tree.bio.ed.ac.uk/software/figtree/) was used to visualize the tree and exported for stylization. Alignment, site filtering, and tree estimation was performed identically and separately for CMT and DCL phylogenies.

\section{Comparative transcriptome analyses}

To estimate the phylogenetic placement of the loss of DCL2 and DCL3 expression, SRA RNA-seq data were downloaded for Landoltia punctata (SRR647050) and Lemna minor (SRR2917879). Data were cleaned and assembled using Trinity v2.5.1 with default options. Assemblies were subject to blastx searches (1e-10) against the present, but not expressed Spirodela DCL3 gene model annotation predicted peptide (Spipo14G0010100).

\section{$\underline{\text { LTR Retrotransposon annotation }}$}


LTR retrotransposons were annotated de novo using GenomeTools LTRharvest with options "-similar 85 -mindistltr 1000 -maxdistltr 15000 -mintsd 5 -maxtsd 20".

\section{Acknowledgements}

We thank the Georgia Advanced Computing Research Center (GACRC) for computational resources. We are grateful to Yinwen Zhang for assistance in interpreting gene body methylation code, and to Brigitte T. Hofmeister for creating and maintaining the browsers. This work was funded by an NSF National Plant Genome Initiative Postdoctoral Fellowship to AH (\#1611853). This study was supported by the National Science Foundation (MCB-1856143) to RJS.

\section{References}

1. M. Lynch, R. Bürger, D. Butcher, W. Gabriel, The mutational meltdown in asexual populations. $J$. Hered. 84, 339-344 (1993).

2. D. J. Crawford, E. Landolt, D. H. Les, R. T. Kimball, Speciation in duckweeds (Lemnaceae): phylogenetic and ecological inferences. Aliso: A Journal of Systematic and Evolutionary Botany 22, 231-242 (2006).

3. W. Wang, et al., The Spirodela polyrhiza genome reveals insights into its neotenous reduction fast growth and aquatic lifestyle. Nat. Commun. 5, 3311 (2014).

4. A. Harkess, et al., A new Spirodela polyrhiza genome and proteome reveal a conserved chromosomal structure with high abundances of proteins favoring energy production. bioRxiv, 2020.01.23.909457 (2020).

5. T. P. Michael, et al., Comprehensive definition of genome features in Spirodela polyrhiza by high-depth physical mapping and short-read DNA sequencing strategies. Plant J. 89, 617-635 (2017).

6. D. An, et al., Plant evolution and environmental adaptation unveiled by long-read whole-genome sequencing of Spirodela. Proc. Natl. Acad. Sci. U. S. A. 116, 18893-18899 (2019).

7. R. S. Dodd, V. Douhovnikoff, Adjusting to Global Change through Clonal Growth and Epigenetic Variation. Front. Ecol. Evol. 4 (2016).

8. C. A. Ibarra, et al., Active DNA demethylation in plant companion cells reinforces transposon methylation in gametes. Science 337 (2012).

9. V. K. Schoft, et al., Function of the DEMETER DNA glycosylase in the Arabidopsis thaliana male gametophyte. Proc. Natl. Acad. Sci. U. S. A. 108 (2011).

10. J. S. Park, et al., Control of DEMETER DNA demethylase gene transcription in male and female gamete companion cells in Arabidopsis thaliana. Proc. Natl. Acad. Sci. U. S. A. 114 (2017).

11. B. T. Hofmeister, K. Lee, N. A. Rohr, D. W. Hall, R. J. Schmitz, Stable inheritance of DNA 
methylation allows creation of epigenotype maps and the study of epiallele inheritance patterns in the absence of genetic variation. Genome Biol. 18, 155 (2017).

12. R. S. Meyer, A. E. DuVal, H. R. Jensen, Patterns and processes in crop domestication: an historical review and quantitative analysis of 203 global food crops. New Phytol. 196, 29-48 (2012).

13. C. E. Niederhuth, et al., Widespread natural variation of DNA methylation within angiosperms. Genome Biol. 17, 194 (2016).

14. E. J. Finnegan, R. K. Genger, W. J. Peacock, E. S. Dennis, DNA METHYLATION IN PLANTS. Annu. Rev. Plant Physiol. Plant Mol. Biol. 49, 223-247 (1998).

15. S. J. Cokus, et al., Shotgun bisulphite sequencing of the Arabidopsis genome reveals DNA methylation patterning. Nature 452, 215-219 (2008).

16. R. Lister, et al., Highly integrated single-base resolution maps of the epigenome in Arabidopsis. Cell 133, 523-536 (2008).

17. J. Du, et al., Dual binding of chromomethylase domains to H3K9me2-containing nucleosomes directs DNA methylation in plants. Cell 151, 167-180 (2012).

18. J. Du, L. M. Johnson, S. E. Jacobsen, D. J. Patel, DNA methylation pathways and their crosstalk with histone methylation. Nat. Rev. Mol. Cell Biol. 16, 519-532 (2015).

19. A. M. Lindroth, et al., Requirement of CHROMOMETHYLASE3 for maintenance of CpXpG methylation. Science 292, 2077-2080 (2001).

20. J. P. Jackson, A. M. Lindroth, X. Cao, S. E. Jacobsen, Control of CpNpG DNA methylation by the KRYPTONITE histone H3 methyltransferase. Nature 416, 556-560 (2002).

21. Q. Gouil, D. C. Baulcombe, DNA Methylation Signatures of the Plant Chromomethyltransferases. PLoS Genet. 12, e1006526 (2016).

22. R. M. Erdmann, C. L. Picard, RNA-directed DNA Methylation. PLoS Genet. 16, e1009034 (2020).

23. R. H. Dowen, et al., Widespread dynamic DNA methylation in response to biotic stress. Proc. Natl. Acad. Sci. U. S. A. 109, E2183-91 (2012).

24. X. Shen, et al., Natural CMT2 Variation Is Associated With Genome-Wide Methylation Changes and Temperature Seasonality. PLoS Genetics 10, e1004842 (2014).

25. T. Kawakatsu, et al., Epigenomic Diversity in a Global Collection of Arabidopsis thaliana Accessions. Cell 166, 492-505 (2016).

26. T. P. Michael, et al., Comprehensive definition of genome features in Spirodela polyrhiza by high-depth physical mapping and short-read DNA sequencing strategies. Plant J. 89, 617-635 (2017).

27. J. M. Noshay, et al., Monitoring the interplay between transposable element families and DNA methylation in maize. PLoS Genet. 15, e1008291 (2019). 
28. M. A. Matzke, R. A. Mosher, RNA-directed DNA methylation: an epigenetic pathway of increasing complexity. Nat. Rev. Genet. 15, 394-408 (2014).

29. L. Ma, et al., Angiosperms Are Unique among Land Plant Lineages in the Occurrence of Key Genes in the RNA-Directed DNA Methylation (RdDM) Pathway. Genome Biol. Evol. 7, 2648-2662 (2015).

30. P. Patel, S. M. Mathioni, R. Hammond, A. E. Harkess, Reproductive phasiRNA loci and DICER-LIKE5, but not microRNA loci, diversified in monocotyledonous plants. bioRxiv (2020).

31. X. Zhang, I. R. Henderson, C. Lu, P. J. Green, S. E. Jacobsen, Role of RNA polymerase IV in plant small RNA metabolism. Proc. Natl. Acad. Sci. U. S. A. 104, 4536-4541 (2007).

32. R. A. Mosher, F. Schwach, D. Studholme, D. C. Baulcombe, PolIVb influences RNA-directed DNA methylation independently of its role in siRNA biogenesis. Proc. Natl. Acad. Sci. U. S. A. 105, 3145-3150 (2008).

33. D. Zilberman, X. Cao, S. E. Jacobsen, ARGONAUTE4 control of locus-specific siRNA accumulation and DNA and histone methylation. Science 299, 716-719 (2003).

34. C. F. Li, et al., An ARGONAUTE4-containing nuclear processing center colocalized with Cajal bodies in Arabidopsis thaliana. Cell 126, 93-106 (2006).

35. P. Fourounjian, et al., Post-transcriptional adaptation of the aquatic plant Spirodela polyrhiza under stress and hormonal stimuli. Plant J. 41, 1 (2019).

36. P. N. T. Hoang, et al., Generating a high-confidence reference genome map of the Greater Duckweed by integration of cytogenomic, optical mapping, and Oxford Nanopore technologies. Plant J. 96, 670-684 (2018).

37. J.-S. Parent, N. Bouteiller, T. Elmayan, H. Vaucheret, Respective contributions of Arabidopsis DCL2 and DCL4 to RNA silencing. The Plant Journal 81, 223-232 (2015).

38. V. Gasciolli, A. C. Mallory, D. P. Bartel, H. Vaucheret, Partially redundant functions of Arabidopsis DICER-like enzymes and a role for DCL4 in producing trans-acting siRNAs. Curr. Biol. 15, 1494-1500 (2005).

39. Z. Xie, E. Allen, A. Wilken, J. C. Carrington, DICER-LIKE 4 functions in trans-acting small interfering RNA biogenesis and vegetative phase change in Arabidopsis thaliana. Proc. Natl. Acad. Sci. U. S. A. 102, 12984-12989 (2005).

40. M. Yoshikawa, A. Peragine, M. Y. Park, R. S. Poethig, A pathway for the biogenesis of trans-acting siRNAs in Arabidopsis. Genes Dev. 19, 2164-2175 (2005).

41. Q. Fei, R. Xia, B. C. Meyers, Phased, secondary, small interfering RNAs in posttranscriptional regulatory networks. Plant Cell 25, 2400-2415 (2013).

42. C. Teng, et al., Dicer-like 5 deficiency confers temperature-sensitive male sterility in maize. bioRxiv, 498410 (2018).

43. Y. Onodera, et al., Plant nuclear RNA polymerase IV mediates siRNA and DNA 
methylation-dependent heterochromatin formation. Cell 120, 613-622 (2005).

44. H. Stroud, et al., Non-CG methylation patterns shape the epigenetic landscape in Arabidopsis. Nat. Struct. Mol. Biol. 21, 64-72 (2014).

45. A. J. Bewick, et al., The evolution of CHROMOMETHYLASES and gene body DNA methylation in plants. Genome Biol. 18, 65 (2017).

46. J. M. Wendte, et al., Epimutations are associated with CHROMOMETHYLASE 3-induced de novo DNA methylation. Elife 8 (2019).

47. M. J. Dubin, et al., DNA methylation in Arabidopsis has a genetic basis and shows evidence of local adaptation. Elife 4, e05255 (2015).

48. A. Zemach, et al., The Arabidopsis nucleosome remodeler DDM1 allows DNA methyltransferases to access H1-containing heterochromatin. Cell 153, 193-205 (2013).

49. H. X. Cao, G. T. H. Vu, W. Wang, J. Messing, I. Schubert, Chromatin organisation in duckweed interphase nuclei in relation to the nuclear DNA content. Plant Biol. 17 Suppl 1, 120-124 (2015).

50. Y. V. Bernatavichute, X. Zhang, S. Cokus, M. Pellegrini, S. E. Jacobsen, Genome-wide association of histone $\mathrm{H} 3$ lysine nine methylation with CHG DNA methylation in Arabidopsis thaliana. PLoS One 3, e3156 (2008).

51. S. Inagaki, T. Kakutani, What triggers differential DNA methylation of genes and TEs: contribution of body methylation? Cold Spring Harb. Symp. Quant. Biol. 77, 155-160 (2012).

52. A. J. Bewick, et al., On the origin and evolutionary consequences of gene body DNA methylation. Proc. Natl. Acad. Sci. U. S. A. 113, 9111-9116 (2016).

53. Y. Zhang, J. M. Wendte, L. Ji, R. J. Schmitz, Natural variation in DNA methylation homeostasis and the emergence of epialleles. Proc. Natl. Acad. Sci. U. S. A. 117, 4874-4884 (2020).

54. R. K. Tran, et al., DNA methylation profiling identifies CG methylation clusters in Arabidopsis genes. Curr. Biol. 15, 154-159 (2005).

55. S. Takuno, J.-H. Ran, B. S. Gaut, Evolutionary patterns of genic DNA methylation vary across land plants. Nature Plants 2 (2016).

56. S. Takuno, D. K. Seymour, B. S. Gaut, The Evolutionary Dynamics of Orthologs That Shift in Gene Body Methylation between Arabidopsis Species. Mol. Biol. Evol. 34, 1479-1491 (2017).

57. C. I. Stoddard, et al., A Nucleosome Bridging Mechanism for Activation of a Maintenance DNA Methyltransferase. Mol. Cell 73, 73-83.e6 (2019).

58. H. Saze, A. Shiraishi, A. Miura, T. Kakutani, Control of genic DNA methylation by a jmjC domain-containing protein in Arabidopsis thaliana. Science 319 (2008).

59. T. Ito, et al., Genome-wide negative feedback drives transgenerational DNA methylation dynamics in Arabidopsis. PLoS Genet. 11, e1005154 (2015). 
60. A. Miura, et al., An Arabidopsis jmjC domain protein protects transcribed genes from DNA methylation at CHG sites. EMBO J. 28 (2009).

61. P. Ziegler, K. Adelmann, S. Zimmer, C. Schmidt, K.-J. Appenroth, Relative in vitro growth rates of duckweeds (Lemnaceae) - the most rapidly growing higher plants. Plant Biol. 17 Suppl 1, 33-41 (2015).

62. E. Landolt, R. Kandeler, Biosystematic investigations in the family of duckweeds (Lemnaceae), Vol. 4: The family of Lemnaceae - a monographic study, Vol. 2 (phytochemistry, physiology, application, bibliography). Veroeffentlichungen des Geobotanischen Instituts der ETH, Stiftung Ruebel (Switzerland) (1987).

63. J. P. Khurana, S. C. Maheshwari, Some effects of salicylic acid on growth and flowering in Spirodela polyrrhiza SP20. Plant Cell Physiol. 21, 923-927 (1980).

64. D. McKey, M. Elias, B. Pujol, A. Duputié, The evolutionary ecology of clonally propagated domesticated plants. New Phytol. 186, 318-332 (2010).

65. E. K. H. Ho, M. Bartkowska, S. I. Wright, A. F. Agrawal, Population genomics of the facultatively asexual duckweed Spirodela polyrhiza. New Phytol. 224, 1361-1371 (2019).

66. S. Xu, et al., Low genetic variation is associated with low mutation rate in the giant duckweed. Nat. Commun. 10, 1243 (2019).

67. E. S. Dolgin, B. Charlesworth, The fate of transposable elements in asexual populations. Genetics 174, 817-827 (2006).

68. M. A. Urich, J. R. Nery, R. Lister, R. J. Schmitz, J. R. Ecker, MethylC-seq library preparation for base-resolution whole-genome bisulfite sequencing. Nat. Protoc. 10, 475-483 (2015).

69. M. D. Schultz, et al., Corrigendum: Human body epigenome maps reveal noncanonical DNA methylation variation. Nature 530, 242 (2016).

70. M. Martin, Cutadapt removes adapter sequences from high-throughput sequencing reads. EMBnet.journal 17, 10 (2011).

71. B. Langmead, C. Trapnell, M. Pop, S. L. Salzberg, Ultrafast and memory-efficient alignment of short DNA sequences to the human genome. Genome Biol. 10, R25 (2009).

72. M. D. Schultz, R. J. Schmitz, J. R. Ecker, "Leveling" the playing field for analyses of single-base resolution DNA methylomes. Trends in Genetics 28, 583-585 (2012).

73. M. Zaratiegui, et al., RNAi promotes heterochromatic silencing through replication-coupled release of RNA Pol II. Nature 479, 135-138 (2011).

74. H. Li, R. Durbin, Fast and accurate short read alignment with Burrows-Wheeler transform. Bioinformatics 25, 1754-1760 (2009).

75. A. R. Quinlan, I. M. Hall, BEDTools: a flexible suite of utilities for comparing genomic features. Bioinformatics 26, 841-842 (2010). 
bioRxiv preprint doi: https://doi.org/10.1101/2020.12.03.410332; this version posted December 3, 2020. The copyright holder for this preprint (which was not certified by peer review) is the author/funder, who has granted bioRxiv a license to display the preprint in perpetuity. It is made available under aCC-BY-NC-ND 4.0 International license.

76. Y. Zhang, et al., Model-based analysis of ChIP-Seq (MACS). Genome Biol. 9, R137 (2008). 